\title{
A TWO STEP NEWTON TYPE ITERATION FOR ILL-POSED HAMMERSTEIN TYPE OPERATOR EQUATIONS IN HILBERT SCALES
}

\author{
MONNANDA ERAPPA SHOBHA, SANTHOSH GEORGE \\ AND M. KUNHANANDAN
}

Communicated by Masahiro Yamamoto

\begin{abstract}
In this paper regularized solutions of ill-posed Hammerstein type operator equation $K F(x)=y$, where $K: X \rightarrow Y$ is a bounded linear operator with non-closed range and $F: X \rightarrow X$ is non-linear, are obtained by a two step Newton type iterative method in Hilbert scales, where the available data is $y^{\delta}$ in place of actual data $y$ with $\left\|y-y^{\delta}\right\| \leq \delta$. We require only a weaker assumption $\left\|F^{\prime}\left(x_{0}\right) x\right\| \sim\|x\|_{-b}$ compared to the usual assumption $\left\|F^{\prime}(\widehat{x}) x\right\| \sim\|x\|_{-b}$, where $\widehat{x}$ is the actual solution of the problem, which is assumed to exist, and $x_{0}$ is the initial approximation. Two cases, viz-aviz, (i) when $F^{\prime}\left(x_{0}\right)$ is boundedly invertible and (ii) $F^{\prime}\left(x_{0}\right)$ is non-invertible but $F$ is monotone operator, are considered. We derive error bounds under certain general source conditions by choosing the regularization parameter by an a priori manner as well as by using a modified form of the adaptive scheme proposed by Perverzev and Schock [14].
\end{abstract}

1. Introduction. In this paper we present an iterative method which combines Tikhonov regularization with the modified Newton's method in Hilbert scales, for approximately solving the operator equation

$$
K F(x)=y .
$$

Here $K: X \rightarrow Y$ is a bounded linear operator with its range $R(K)$ not closed in $X, F: D(F) \subseteq X \rightarrow X$ is a nonlinear operator, where $D(F)$

2010 AMS Mathematics subject classification. Primary 65J15, 65J20, 47H17, $47 \mathrm{~A} 50$.

Keywords and phrases. Ill-posed problems, Tikhonov regularization, Hilbert scales, Newton's method, adaptive choice.

The first author was supported by the National Institute of Technology Karnataka, India.

Received by the editors on July 18, 2012, and in revised form on March 25, 2013.

DOI:10.1216/JIE-2014-26-1-91 Copyright (c)2014 Rocky Mountain Mathematics Consortium 
is the domain of $F$, and $X$ and $Y$ are Hilbert spaces. We shall use the notations $\langle\cdot, \cdot\rangle$ and $\|\cdot\|$ for the inner product and the corresponding norm in the Hilbert spaces. The equation (1.1) is, in general, ill-posed, in the sense that a unique solution that depends continuously on the data does not exist and hence need to be regularized to get stable approximate solution. Further, we assume that only approximate data $y^{\delta}$ satisfying

$$
\left\|y-y^{\delta}\right\| \leq \delta
$$

is available.

A typical example of equation (1.1) is the classical Hammerstein operator equation (see [9, page 430])

$$
\int_{0}^{1} k(s, t) h(s, x(s)) d s=y(t), \quad t \in[0,1],
$$

where $k(\cdot, \cdot)$ is a non-degenerate kernel which is square integrable, that is,

$$
\int_{0}^{1} \int_{0}^{1}|k(s, t)|^{2} d s d t<\infty
$$

and $h:[0,1] \times[0,1] \rightarrow \mathbf{R}$ is a suitable function. Then equation (1.3) takes the form (1.1) with $K: L^{2}[0,1] \rightarrow L^{2}[0,1]$ defined by

$$
K u(t)=\int_{0}^{1} k(s, t) u(s) d s, \quad t \in[0,1]
$$

and $F: L^{2}[0,1] \rightarrow L^{2}[0,1]$ is the nonlinear 'superposition operator' defined by

$$
F(x)(s)=h(s, x(s)), \quad s \in[0,1] .
$$

Note that, due to the nonlinearity of $F$, the solution of (1.1), even when it exists, need not be unique. Therefore, one applies some selection criteria. Following the definition in $[\mathbf{5 , 6}]$, a solution $\widehat{x} \in D(F)$ of (1.1) is called an $x_{0}$-minimum norm solution $\left(x_{0}\right.$-MNS) of $(1.1)$, if (1.4)

$$
\left\|F(\widehat{x})-F\left(x_{0}\right)\right\|=\min \left\{\left\|F(x)-F\left(x_{0}\right)\right\|: K F(x)=y, x \in D(F)\right\} .
$$

In the following, we always assume the existence of an $x_{0}$-MNS for exact data $y$, i.e.,

$$
K F(\widehat{x})=y .
$$


One may observe that equation (1.1) is equivalent to

$$
K\left[F(x)-F\left(x_{0}\right)\right]=y-K F\left(x_{0}\right)
$$

for a given $x_{0}$, so that the solution $\widehat{x}$ of (1.1) is obtained by solving

$$
K z=y-K F\left(x_{0}\right)
$$

for $z$ and then solving the non-linear equation

$$
F(x)=z+F\left(x_{0}\right)
$$

An advantage of approximately solving (1.5) and (1.6) to obtain an approximate solution for (1.1) is that one can use any regularization method for linear ill-posed equations for solving (1.5) and any method for solving non-linear equation (1.6). This advantage was exploited by the second author and his collaborators (see $[\mathbf{3 - 5}, \mathbf{8}]$ ). In $[\mathbf{2 1}]$, Shobha et al. considered a two step iterative method for solving (1.1) in a Hilbert space.

In order to improve the rate of convergence many authors have considered the Hilbert scale variant of the regularization methods for solving ill-posed operator equations, for example, $[\mathbf{1}, \mathbf{1 0 - 1 3}, \mathbf{1 5 - 1 7}]$. In this paper, we consider the Hilbert scale variant of the method considered in [21]. For analyzing (1.1) in the setting of Hilbert scales, we consider a Hilbert scale $\left\{X_{t}\right\}_{t \in \mathbf{R}}$ generated by a strictly positive operator $L: D(L) \rightarrow X$ with $D(L)$ dense in $X$ satisfying

$$
\|L x\| \geq\|x\|, \quad x \in D(L) .
$$

Recall $([\mathbf{1 5}, \mathbf{2 2}])$ that the space $X_{t}$ is the completion of $D:=$ $\cap_{k=0}^{\infty} D\left(L^{k}\right)$ with respect to the norm $\|x\|_{t}$, induced by the inner product

$$
\left\langle x_{1}, x_{2}\right\rangle_{t}=\left\langle L^{t / 2} x_{1}, L^{t / 2} x_{2}\right\rangle
$$

i.e.,

$$
\|x\|_{t}=\left\|L^{t / 2} x\right\|, \quad t \in \mathbf{R} .
$$

As in [21], we consider two cases of the operator $F$ in (1.1).

Case I. $F^{\prime}\left(x_{0}\right)^{-1}$ exists and is bounded. Thus, the ill-posedness of (1.1) is essentially due to the non-closedness of the range of the linear 
operator $K$, as $F^{\prime}\left(x_{0}\right)^{-1}$ is a bounded linear operator implies equation (1.6) is well-posed (see [18, page 26]). In this case, we consider the sequence $\left(x_{n, \alpha, s}^{\delta}\right)$ defined iteratively by

$$
y_{n, \alpha, s}^{\delta}=x_{n, \alpha, s}^{\delta}-F^{\prime}\left(x_{0}\right)^{-1}\left[F\left(x_{n, \alpha, s}^{\delta}\right)-z_{\alpha, s}^{\delta}\right]
$$

and

$$
x_{n+1, \alpha, s}^{\delta}=y_{n, \alpha, s}^{\delta}-F^{\prime}\left(x_{0}\right)^{-1}\left[F\left(y_{n, \alpha, s}^{\delta}\right)-z_{\alpha, s}^{\delta}\right]
$$

where $x_{0, \alpha, s}^{\delta}:=x_{0}$ is the initial approximation for the solution $\widehat{x}$ of (1.1),

$$
z_{\alpha, s}^{\delta}:=F\left(x_{0}\right)+\left(L^{-s} K^{*} K+\alpha I\right)^{-1} L^{-s} K^{*}\left(y^{\delta}-K F\left(x_{0}\right)\right)
$$

and $\alpha$ is the regularization parameter to be chosen appropriately from the finite set $D_{N}:=\left\{\alpha_{i}: 0<\alpha_{0}<\alpha_{1}<\cdots<\alpha_{N}\right\}$ depending on the inexact data $y^{\delta}$ and the error level $\delta$ satisfying (1.2). We use the adaptive parameter selection procedure suggested by Pereverzev and Schock [14] for the selection of regularization parameters.

Example 1.1. Consider the non-linear Hammerstein operator equation

$$
\int_{\Omega} k(s, t) f_{\lambda}(t, x(t)) d t=y(s)
$$

with $\Omega \subset \mathbf{R}$ a bounded domain, $k(s, t): \sum_{n=0}^{\infty}(n+1)^{-2} u_{n}(s) u_{n}(t)$, where $u_{n}(s)=\sqrt{2} \cos (2 n \pi s)$ and $f_{\lambda}: \Omega \times \mathbf{R} \rightarrow \mathbf{R}$ is defined as $f_{\lambda}(s, x)=b(s) g(x(s))+\lambda c(s)$, where $0 \neq c \in L^{p}, 0<b \in L^{p /(p-q)}$ for some $q \in(2, p)$ (cf. [2]) and $g$ is a differentiable function such that $g^{\prime}\left(x_{0}(t)\right)>\kappa>0$, for all $t \in \Omega$.

Note that (1.10) is of the form $K F(x)=y$, where $K: L^{2}(\Omega) \rightarrow L^{2}(\Omega)$ is given by

$$
K(x(s))=\int_{\Omega} k(s, t) x(t) d t
$$

and $F: L^{2}(\Omega) \rightarrow L^{2}(\Omega)$ is given by

$$
F(x(s))=f_{\lambda}(s, x(s)) .
$$


Observe that

$$
F^{\prime}(x) h(s)=f_{\lambda x}(s, x(s)) h(s)=b(s) g^{\prime}(x(s)) h(s),
$$

for $x, y \in B_{R}\left(x_{0}\right)$ and $h \in L^{2}(\Omega)$. Further note that, since $g^{\prime}\left(x_{0}(t)\right)>$ $\kappa>0$, for all $t \in \Omega, F^{\prime}\left(x_{0}\right)^{-1}=1 /\left(b(s) g^{\prime}\left(x_{0}\right)\right)$ exists and is a bounded operator.

Case II. $F^{\prime}\left(x_{0}\right)$ is non-invertible, but $F$ is a monotone operator. Recall that $([\mathbf{2 0}, \mathbf{2 3}])$ the operator $F$ is said to be a monotone operator if $\langle F(x)-F(y), x-y\rangle \geq 0$, for all $x, y \in D(F)$. In this case, we consider the sequence $\left(\widetilde{x}_{n, \alpha, s}^{\delta}\right)$ defined iteratively by

$$
\begin{aligned}
\widetilde{y}_{n, \alpha, s}^{\delta}= & \widetilde{x}_{n, \alpha, s}^{\delta}-\left(F^{\prime}\left(x_{0}\right)+\frac{\alpha}{c} L^{s / 2}\right)^{-1} \\
& \times\left[F\left(\widetilde{x}_{n, \alpha, s}^{\delta}\right)-z_{\alpha, s}^{\delta}+\frac{\alpha}{c} L^{s / 2}\left(\widetilde{x}_{n, \alpha, s}^{\delta}-x_{0}\right)\right]
\end{aligned}
$$

and

$$
\begin{aligned}
\widetilde{x}_{n+1, \alpha}^{\delta}= & \widetilde{y}_{n, \alpha, s}^{\delta}-\left(F^{\prime}\left(x_{0}\right)+\frac{\alpha}{c} L^{s / 2}\right)^{-1} \\
& \times\left[F\left(\widetilde{y}_{n, \alpha, s}^{\delta}\right)-z_{\alpha, s}^{\delta}+\frac{\alpha}{c} L^{s / 2}\left(\widetilde{y}_{n, \alpha, s}^{\delta}-x_{0}\right)\right]
\end{aligned}
$$

where $\widetilde{x}_{0, \alpha, s}^{\delta}:=x_{0}$, with $x_{0}, z_{\alpha, s}^{\delta}$ and $\alpha$ as in Case I and $0<c \leq \alpha$.

Example 1.2. In this example, we consider the operator $K F$ : $L^{2}(0,1) \rightarrow L^{2}(0,1)$ where $K: L^{2}(0,1) \rightarrow L^{2}(0,1)$ is defined by

$$
K(x)(t)=\int_{0}^{1} k(t, s) x(s) d s
$$

and $F: D(F) \subseteq L^{2}(0,1) \rightarrow L^{2}(0,1)$ is defined by

$$
F(u):=\int_{0}^{1} k(t, s) u^{3}(s) d s
$$


where

$$
k(t, s)= \begin{cases}(1-t) s & 0 \leq s \leq t \leq 1 \\ (1-s) t & 0 \leq t \leq s \leq 1 .\end{cases}
$$

Then, for all $x(t), y(t): x(t)>y(t)$, (see [20, subsection 4.3])

$$
\langle F(x)-F(y), x-y\rangle=\int_{0}^{1}\left[\int_{0}^{1} k(t, s)\left(x^{3}-y^{3}\right)(s) d s\right](x-y)(t) d t \geq 0 .
$$

Thus, the operator $F$ is monotone.

The paper is organized as follows. In Section 2, we give preliminaries and the adaptive scheme for choosing the regularization parameter $\alpha$ for Tikhonov regularization of (1.5) in the setting of Hilbert scales. The proposed method and the error estimates for the case when $F^{\prime}\left(x_{0}\right)$ is invertible are given in subsection 3.1, and the case when $F^{\prime}\left(x_{0}\right)$ is noninvertible but $F$ is monotone is given in subsection 3.2. We conclude the paper in Section 4.

2. Preliminaries. We assume that the ill-posed nature of the operator $K$ is related to the Hilbert scale $\left\{X_{t}\right\}_{t \in \mathbf{R}}$ according to the relation

$$
c_{1}\|x\|_{-a} \leq\|K x\| \leq c_{2}\|x\|_{-a}, \quad x \in X,
$$

for some reals $a, c_{1}$ and $c_{2}$.

Observe that from the relation $\langle K x, y\rangle=\left\langle x, K^{*} y\right\rangle=\left\langle x, L^{-s} K^{*} y\right\rangle_{s}$ for all $x \in X$ and $y \in Y$, we conclude that $L^{-s} K^{*}: Y \rightarrow X$ is the adjoint of the operator $K$ in $X$. Consequently, $L^{-s} K^{*} K: X \rightarrow X$ is self-adjoint. Further we note that

$$
\left(A_{s}^{*} A_{s}+\alpha I\right)^{-1} L^{s / 2}=L^{s / 2}\left(L^{-s} K^{*} K+\alpha I\right)^{-1}
$$

where $A_{s}=K L^{-s / 2}$.

One of the crucial results for proving the results in this paper is the following proposition, where $f$ and $g$ are defined by

$$
f(t)=\min \left\{c_{1}^{t}, c_{2}^{t}\right\}, \quad g(t)=\max \left\{c_{1}^{t}, c_{2}^{t}\right\}, \quad t \in \mathbf{R},|t| \leq 1 .
$$

Proposition 2.1 (see [24, Proposition 2.1]). For $s \geq 0$ and $|\nu| \leq 1$,

$$
f(\nu)\|x\|_{-\nu(s+a)} \leq\left\|\left(A_{s}^{*} A_{s}\right)^{\nu / 2} x\right\| \leq g(\nu)\|x\|_{-\nu(s+a)}, \quad x \in H .
$$


We make use of the relation

$$
\left\|\left(A_{s}+\alpha I\right)^{-1} A_{s}^{p}\right\| \leq \alpha^{p-1}, \quad p>0,0<p \leq 1,
$$

which follows from the spectral properties of the positive self-adjoint operator $A_{s}, s>0$.

The following assumption on source condition is based on a source function $\varphi$ and a property of the source function $\varphi$. We will be using this assumption to obtain an error estimate for $\left\|z_{\alpha, s}^{\delta}-F(\widehat{x})\right\|$.

Assumption 2.2. There exists a continuous, strictly monotonically increasing function $\varphi:\left(0,\left\|A_{s}^{*} A_{s}\right\|\right] \rightarrow(0, \infty)$ such that the following conditions hold:

- $\lim _{\lambda \rightarrow 0} \varphi(\lambda)=0$,

$$
\sup _{\lambda>0} \frac{\alpha \varphi(\lambda)}{\lambda+\alpha} \leq \varphi(\alpha) \quad \text { for all } \lambda \in\left(0,\left\|A_{s}^{*} A_{s}\right\|\right]
$$

and

- there exists $v \in X$ with $\|v\| \leq \bar{E}, \bar{E}>0$ such that

$$
\left(A_{s}^{*} A_{s}\right)^{s / 2(s+a)} L^{s / 2}\left(F(\hat{x})-F\left(x_{0}\right)\right)=\varphi\left(A_{s}^{*} A_{s}\right) v .
$$

Remark 2.3. Note that if $F(\widehat{x})-F\left(x_{0}\right) \in X_{t}$, i.e., $\left\|F(\widehat{x})-F\left(x_{0}\right)\right\|_{t} \leq$ $E$, for some $0<t \leq 2 s+a$, then the above assumption is satisfied. This can be seen as follows.

$$
\begin{aligned}
& \left(A_{s}^{*} A_{s}\right)^{s / 2(s+a)} L^{s / 2}\left(F(\widehat{x})-F\left(x_{0}\right)\right) \\
= & \left(A_{s}^{*} A_{s}\right)^{t / 2(s+a)}\left(A_{s}^{*} A_{s}\right)^{(s-t) /(2 s+2 a)} L^{s / 2}\left(F(\widehat{x})-F\left(x_{0}\right)\right)=\varphi\left(A_{s}^{*} A_{s}\right) v,
\end{aligned}
$$

where $\varphi(\lambda)=\lambda^{t / 2(s+a)}$ and $v=\left(A_{s}^{*} A_{s}\right)^{(s-t) /(2 s+2 a)} L^{s / 2}\left(F(\widehat{x})-F\left(x_{0}\right)\right)$.

Further note that

$$
\begin{aligned}
\|v\| & \leq g\left(\frac{s-t}{s+a}\right)\left\|L^{s / 2}\left(F(\widehat{x})-F\left(x_{0}\right)\right)\right\|_{t-s} \\
& \leq g\left(\frac{s-t}{s+a}\right)\left\|\left(F(\widehat{x})-F\left(x_{0}\right)\right)\right\|_{t} \leq \bar{E},
\end{aligned}
$$

where $\bar{E}=g(s-t / s+a) E$. 
Theorem 2.4. Suppose that Assumption 2.2 holds, and let $z_{\alpha, s}:=$ $z_{\alpha, s}^{0}$. Then

1. $\left\|z_{\alpha, s}^{\delta}-z_{\alpha, s}\right\| \leq \psi(s) \alpha^{-a /[2(s+a)]} \delta$,

2. $\left\|F(\widehat{x})-z_{\alpha, s}\right\| \leq \phi(s) \varphi(\alpha)$,

where $\psi(s)=1 / f(s /(s+a)), \phi(s)=\bar{E} / f(s /(s+a))$ and $\psi_{1}(s)=$ $g(s /(s+a)) / f(s /(s+a))$.

Proof. Note that

$$
\begin{aligned}
\left\|z_{\alpha, s}^{\delta}-z_{\alpha, s}\right\| & =\left\|\left(L^{-s} K^{*} K+\alpha I\right)^{-1} L^{-s} K^{*}\left(y^{\delta}-y\right)\right\| \\
& =\left\|L^{-s / 2}\left(A_{s}^{*} A_{s}+\alpha I\right)^{-1} A_{s}^{*}\left(y^{\delta}-y\right)\right\|
\end{aligned}
$$

now by taking $\nu=s /(s+a)$ and $x=\left(A_{s}^{*} A_{s}+\alpha I\right)^{-1} A_{s}^{*}\left(y^{\delta}-y\right)$ in Proposition 2.1, we have

$$
\begin{aligned}
& \left\|z_{\alpha, s}^{\delta}-z_{\alpha, s}\right\| \\
\leq & \frac{1}{f(s /(s+a))}\left\|\left(A_{s}^{*} A_{s}\right)^{s /(2(s+a))}\left(A_{s}^{*} A_{s}+\alpha I\right)^{-1} A_{s}^{*}\left(y^{\delta}-y\right)\right\| \\
= & \frac{1}{f(s /(s+a))}\left\|\left(A_{s}^{*} A_{s}+\alpha I\right)^{-1}\left(A_{s}^{*} A_{s}\right)^{(2 s+a) /(2(s+a))}\left(y^{\delta}-y\right)\right\| \\
\leq & \frac{1}{f(s /(s+a))}\left\|\left(A_{s}^{*} A_{s}+\alpha I\right)^{-1}\left(A_{s}^{*} A_{s}\right)^{(2 s+a) /(2(s+a))}\right\| \delta .
\end{aligned}
$$

We note that relation $(2.13)$ with $p=(2 s+a) /(2(s+a))$ gives

$$
\left\|\left(A_{s}^{*} A_{s}+\alpha I\right)^{-1}\left(A_{s}^{*} A_{s}\right)^{(2 s+a) /(2(s+a))}\right\| \leq \alpha^{-a /(2(s+a))} .
$$

Now (2.14) follows from (2.17) and (2.18). Further, we observe that

$$
\begin{aligned}
&\left\|z_{\alpha, s}-F(\widehat{x})\right\|=\left\|\left[\left(L^{-s} K^{*} K+\alpha I\right)^{-1} L^{-s} K^{*} K-I\right]\left(F(\widehat{x})-F\left(x_{0}\right)\right)\right\| \\
&=\left\|\alpha L^{-s / 2}\left(A_{s}^{*} A_{s}+\alpha I\right)^{-1} L^{s / 2}\left(F(\widehat{x})-F\left(x_{0}\right)\right)\right\| \\
& \leq \frac{1}{f(s / 2(s+a))} \| \alpha\left(A_{s}^{*} A_{s}\right)^{s /(2(s+a))}\left(A_{s}^{*} A_{s}+\alpha I\right)^{-1} \\
& L^{s / 2}\left(F(\widehat{x})-F\left(x_{0}\right)\right) \|
\end{aligned}
$$




$$
\begin{aligned}
& \leq \frac{1}{f(s / 2(s+a))} \| \alpha\left(A_{s}^{*} A_{s}+\alpha I\right)^{-1}\left(A_{s}^{*} A_{s}\right)^{s /(2(s+a))} \\
& L^{s / 2}\left(F(\widehat{x})-F\left(x_{0}\right)\right) \| \\
& =\frac{1}{f(s / 2(s+a))}\left\|\left(A_{s}^{*} A_{s}+\alpha I\right)^{-1} \varphi\left(A_{s}^{*} A_{s}\right) v\right\| .
\end{aligned}
$$

So, by Assumption 2.2, we have that

$$
\left\|z_{\alpha, s}-F(\widehat{x})\right\| \leq \frac{1}{f(s /(s+a))} \varphi(\alpha) \bar{E} .
$$

Again,

$$
\begin{aligned}
\left\|z_{\alpha, s}-F\left(x_{0}\right)\right\| & =\left\|\left(L^{-s} K^{*} K+\alpha I\right)^{-1} L^{-s} K^{*} K\left(F(\widehat{x})-F\left(x_{0}\right)\right)\right\| \\
& =\left\|L^{-s / 2}\left(A_{s}^{*} A_{s}+\alpha I\right)^{-1} A_{s}^{*} A_{s} L^{s / 2}\left(F(\widehat{x})-F\left(x_{0}\right)\right)\right\| \\
& \leq \frac{1}{f(s /(s+a))} \|\left(A_{s}^{*} A_{s}\right)^{s /(2(s+a))}\left(A_{s}^{*} A_{s}+\alpha I\right)^{-1} \\
& \left(A_{s}^{*} A_{s}\right) L^{s / 2}\left(F(\widehat{x})-F\left(x_{0}\right)\right) \| \\
& =\frac{1}{f(s /(s+a))}\left\|\left(A_{s}^{*} A_{s}+\alpha I\right)^{-1}\left(A_{s}^{*} A_{s}\right)\right\| \\
& \left\|\left(A_{s}^{*} A_{s}\right)^{s /(2(s+a))} L^{s / 2}\left(F(\widehat{x})-F\left(x_{0}\right)\right)\right\| \\
& \leq \frac{g(s /(s+a))}{f(s /(s+a))}\left\|L^{s / 2}\left(F(\widehat{x})-F\left(x_{0}\right)\right)\right\|_{-s} \\
& \leq \psi_{1}(s)\left\|F(\widehat{x})-F\left(x_{0}\right)\right\| .
\end{aligned}
$$

This completes the proof of Theorem 2.4.

2.1. Error bounds and parameter choice in Hilbert scales. Let $C_{s}=\max \{\phi(s), \psi(s)\}$. Then, by (2.14), (2.15) and triangle inequality, we have

$$
\left\|F(\widehat{x})-z_{\alpha, s}^{\delta}\right\| \leq C_{s}\left(\varphi(\alpha)+\alpha^{-a /(2(s+a))} \delta\right) .
$$

The error estimate $\varphi(\alpha)+\alpha^{-a /(2(s+a))} \delta$ in (2.19) attains minimum for the choice $\alpha:=\alpha(\delta, s, a)$ which satisfies $\varphi(\alpha)=\alpha^{-a /(2(s+a))} \delta$. Clearly $\alpha(\delta, s, a)=\varphi^{-1}\left(\psi_{s, a}^{-1}(\delta)\right)$, where

$$
\psi_{s, a}(\lambda)=\lambda\left[\varphi^{-1}(\lambda)\right]^{a /(2(s+a))}, \quad 0<\lambda \leq\left\|A_{s}\right\|^{2},
$$


and in this case

$$
\left\|F(\widehat{x})-z_{\alpha, s}^{\delta}\right\| \leq 2 C_{s} \psi_{s, a}^{-1}(\delta),
$$

which has at least optimal order with respect to $\delta, s$ and $a$ (cf. [14]).

2.2. Adaptive scheme and stopping rule. In this paper we consider the adaptive scheme suggested by Pereverzev and Schock in [14], modified suitably, for choosing the parameter $\alpha$ which does not involve even the regularization method in an explicit manner.

Let $i \in\{0,1,2, \ldots, N\}$ and $\alpha_{i}=\mu^{i} \alpha_{0}$ where $\mu=\eta^{2(1+s / a)}, \eta>1$, and $\alpha_{0}=\delta^{2(1+s / a)}$. Let

$$
l:=\max \left\{i: \varphi\left(\alpha_{i}\right) \leq \alpha_{i}^{-a /(2(s+a))} \delta\right\}<N
$$

and

$$
k:=\max \left\{i:\left\|z_{\alpha_{i, s}}^{\delta}-z_{\alpha_{j, s}}^{\delta}\right\| \leq 4 \alpha_{j}^{-a /(2(s+a))} \delta, j=0,1,2, \ldots, i-1\right\} .
$$

Analogous to the proof of Theorem 4.3 in [5], we have the following theorem.

Theorem 2.5 (cf. [5, Theorem 4.3]). Let $l$ be as in (2.21), $k$ as in (2.22), $\psi_{s, a}$ as in (2.20) and $z_{\alpha_{k}, s}^{\delta}$ as in (1.9) with $\alpha=\alpha_{k}$. Then $l \leq k$ and

$$
\left\|F(\widehat{x})-z_{\alpha_{k}, s}^{\delta}\right\| \leq C_{s}\left(2+\frac{4 \eta}{\eta-1}\right) \eta \psi_{s, a}^{-1}(\delta),
$$

where $C_{s}$ is as in (2.19).

3. The method and convergence analysis. We will be using the following assumption to prove Theorems 3.3 and 3.11 .

Assumption 3.1 (cf. [20, Assumption 3 (A3)]). There exists a constant $k_{0} \geq 0$ such that, for every $x, u \in D(F)$ and $v \in X$, there exists an element $\Phi(x, u, v) \in X$ such that $\left[F^{\prime}(x)-F^{\prime}(u)\right] v=$ $F^{\prime}(u) \Phi(x, u, v),\|\Phi(x, u, v)\| \leq k_{0}\|v\|\|x-u\|$.

\section{1.}

Case I: $F\left(x_{0}\right)^{-1}$ is a bounded linear operator. Consider the two step iterative method defined as in (1.7) and (1.8) with $\alpha_{k}$ in place of $\alpha$. 
We assume that $F$ possesses a uniformly bounded Fréchet derivative for all $x \in D(F)$, i.e., $\left\|F^{\prime}(x)\right\| \leq M$, for all $x$ in a neighborhood of $x_{0}$ and for some $M>0$, and $\left\|F^{\prime}\left(x_{0}\right)^{-1}\right\|:=\beta, \beta>0$. Let

$$
e_{n, \alpha_{k}, s}^{\delta}:=\left\|y_{n, \alpha_{k}, s}^{\delta}-x_{n, \alpha_{k}, s}^{\delta}\right\|, \quad \text { for all } n \geq 0,
$$

and let $\delta_{0}<1 /\left(4 k_{0} \beta \psi(s)\right) \alpha_{0}^{a /(2(s+a))}$ and $\left\|\widehat{x}-x_{0}\right\| \leq \rho$, with

$$
\rho<\frac{1}{\psi_{1}(s) M}\left[\frac{1}{4 k_{0} \beta}-\psi(s) \alpha_{0}^{-a /(2(s+a))} \delta_{0}\right]
$$

and

$$
\gamma_{\rho}:=\beta\left[\psi_{1}(s) M \rho+\psi(s) \alpha^{-a /(2(s+a))} \delta\right] .
$$

Further, let

$$
r_{1}=\frac{1-\sqrt{1-4 k_{0} \gamma_{\rho}}}{2 k_{0}}
$$

and

$$
r_{2}=\min \left\{\frac{1}{k_{0}}, \frac{1+\sqrt{1-4 k_{0} \gamma_{\rho}}}{2 k_{0}}\right\} .
$$

For $r \in\left(r_{1}, r_{2}\right)$, let

$$
q=k_{0} r
$$

Then $q<1$.

Lemma 3.2. Let $e_{0, \alpha_{k}, s}^{\delta}$ be as in (3.23). Then $e_{0, \alpha_{k}, s}^{\delta} \leq \gamma_{\rho}$.

Proof. Observe that

$$
\begin{aligned}
e_{0, \alpha_{k}, s}^{\delta}= & \left\|y_{0, \alpha_{k}, s}^{\delta}-x_{0, \alpha_{k}, s}^{\delta}\right\| \\
= & \left\|F^{\prime}\left(x_{0}\right)^{-1}\left(F\left(x_{0}\right)-z_{\alpha_{k}, s}^{\delta}\right)\right\| \\
\leq & \beta\left\|F\left(x_{0}\right)-z_{\alpha_{k}, s}^{\delta}\right\| \\
\leq & \beta\left[\left\|F\left(x_{0}\right)-z_{\alpha_{k}, s}\right\|\right. \\
& \left.+\left\|z_{\alpha_{k}, s}-z_{\alpha_{k}, s}^{\delta}\right\|\right] .
\end{aligned}
$$


Now, using (2.14) and (2.16) in (3.25), one can see that

$$
\begin{aligned}
e_{0, \alpha_{k}, s}^{\delta} & \leq \beta\left[\psi_{1}(s)\left\|F(\widehat{x})-F\left(x_{0}\right)\right\|+\psi(s) \alpha^{-a /(2(s+a))} \delta\right] \\
& \leq \beta\left[\psi_{1}(s) M \rho+\psi(s) \alpha^{-a /(2(s+a))} \delta\right]=\gamma_{\rho} .
\end{aligned}
$$

This completes the proof.

Theorem 3.3. Let $e_{n, \alpha_{k}, s}^{\delta}$ and $q$ be as in equation (3.23) and (3.24), respectively, $y_{n, \alpha_{k}, s}^{\delta}$ and $x_{n, \alpha_{k}, s}^{\delta}$ defined as in (1.7) and (1.8), respectively, with $\alpha=\alpha_{k}$ and $\delta \in\left(0, \delta_{0}\right]$. Then, under Assumption 3.1 and Lemma 3.2, $x_{n, \alpha_{k}, s}^{\delta}, y_{n, \alpha_{k}, s}^{\delta} \in B_{r}\left(x_{0}\right)$ and the following estimates hold for all $n \geq 0$ :

(a) $\left\|x_{n, \alpha_{k}, s}^{\delta}-y_{n-1, \alpha_{k}, s}^{\delta}\right\| \leq q\left\|y_{n-1, \alpha_{k}, s}^{\delta}-x_{n-1, \alpha_{k}, s}^{\delta}\right\|$;

(b) $\left\|x_{n, \alpha_{k}, s}^{\delta}-x_{n-1, \alpha_{k}, s}^{\delta}\right\| \leq(1+q)\left\|y_{n-1, \alpha_{k}, s}^{\delta}-x_{n-1, \alpha_{k}, s}^{\delta}\right\|$;

(c) $\left\|y_{n, \alpha_{k}, s}^{\delta}-x_{n, \alpha_{k}, s}^{\delta}\right\| \leq q^{2}\left\|y_{n-1, \alpha_{k}, s}^{\delta}-x_{n-1, \alpha_{k}, s}^{\delta}\right\|$;

(d) $e_{n, \alpha_{k}, s}^{\delta} \leq q^{2 n} \gamma_{\rho}$, for all $n \geq 0$.

Proof. Suppose $x_{n, \alpha_{k}, s}^{\delta}, y_{n, \alpha_{k}, s}^{\delta} \in B_{r}\left(x_{0}\right)$. Then

$$
\begin{aligned}
x_{n+1, \alpha_{k}, s}^{\delta}- & y_{n, \alpha_{k}, s}^{\delta} \\
= & y_{n, \alpha_{k}, s}^{\delta}-x_{n, \alpha_{k}, s}^{\delta}+F^{\prime}\left(x_{0}\right)^{-1}\left[F\left(x_{n, \alpha_{k}, s}^{\delta}\right)-\left(F\left(y_{n, \alpha_{k}, s}^{\delta}\right)\right]\right. \\
= & F^{\prime}\left(x_{0}\right)^{-1}\left[F^{\prime}\left(x_{0}\right)\left(y_{n, \alpha_{k}, s}^{\delta}-x_{n, \alpha_{k}, s}^{\delta}\right)\right. \\
& \left.-\left(F\left(y_{n, \alpha_{k}, s}^{\delta}\right)-F\left(x_{n, \alpha_{k}, s}^{\delta}\right)\right)\right],
\end{aligned}
$$

and hence, by Assumption 3.1, we have

$$
\begin{aligned}
\left\|x_{n+1, \alpha_{k}, s}^{\delta}-y_{n, \alpha_{k}, s}^{\delta}\right\|= & \| F^{\prime}\left(x_{0}\right)^{-1} \int_{0}^{1} F^{\prime}\left(x_{0}\right) \Phi\left(x_{0}, x_{n, \alpha_{k}, s}^{\delta}\right. \\
& \left.+t\left(y_{n, \alpha_{k}, s}^{\delta}-x_{n, \alpha_{k}, s}^{\delta}\right), y_{n, \alpha_{k}, s}^{\delta}-x_{n, \alpha_{k}, s}^{\delta}\right) d t \| \\
\leq & k_{0} r\left\|y_{n, \alpha_{k}, s}^{\delta}-x_{n, \alpha_{k}, s}^{\delta}\right\| .
\end{aligned}
$$

This proves (a). Now (b) follows from (a) and the triangle inequality: $\left\|x_{n, \alpha_{k}, s}^{\delta}-x_{n-1, \alpha_{k}, s}^{\delta}\right\| \leq\left\|x_{n, \alpha_{k}, s}^{\delta}-y_{n-1, \alpha_{k}, s}^{\delta}\right\|+\left\|y_{n-1, \alpha_{k}, s}^{\delta}-x_{n-1, \alpha_{k}, s}^{\delta}\right\|$. 
Again, (c) follows from (a), Assumption 3.1 and the following expression.

$$
\begin{aligned}
e_{n, \alpha_{k}, s}^{\delta}=\| F^{\prime}\left(x_{0}\right)^{-1} \int_{0}^{1}\left[F^{\prime}\left(x_{0}\right)-F^{\prime}\left(x_{n, \alpha_{k}, s}^{\delta}\right.\right. & \left.\left.+t\left(x_{n, \alpha_{k}, s}^{\delta}-y_{n-1, \alpha_{k}, s}^{\delta}\right)\right)\right] \\
& \times\left(x_{n, \alpha_{k}, s}^{\delta}-y_{n-1, \alpha_{k}, s}^{\delta}\right) d t \|
\end{aligned}
$$

and (d) follows from (c). Now we show that $x_{n, \alpha_{k}, s}^{\delta}, y_{n, \alpha_{k}, s}^{\delta} \in B_{r}\left(x_{0}\right)$ by induction.

Note that, by (b) and Lemma 3.2,

$$
\begin{aligned}
\left\|x_{1, \alpha_{k}, s}^{\delta}-x_{0}\right\| & \leq(1+q) e_{0, \alpha_{k}, s}^{\delta} \leq \frac{e_{0, \alpha_{k}, s}^{\delta}}{1-q} \\
& \leq \frac{\gamma_{\rho}}{1-q}<r
\end{aligned}
$$

i.e., $x_{1, \alpha_{k}, s}^{\delta} \in B_{r}\left(x_{0}\right)$. Again, note that, by (3.26) and (c), we have

$$
\begin{aligned}
\left\|y_{1, \alpha_{k}, s}^{\delta}-x_{0}\right\| & \leq\left\|y_{1, \alpha_{k}, s}^{\delta}-x_{1, \alpha_{k}, s}^{\delta}\right\|+\left\|x_{1, \alpha_{k}, s}^{\delta}-x_{0}\right\| \\
& \leq\left(1+q+q^{2}\right) e_{0, \alpha_{k}, s}^{\delta} \\
& \leq \frac{\gamma_{\rho}}{1-q}<r,
\end{aligned}
$$

i.e., $y_{1, \alpha_{k}, s}^{\delta} \in B_{r}\left(x_{0}\right)$. Further, let us assume that $x_{m, \alpha_{k}, s}^{\delta}, y_{m, \alpha_{k}, s}^{\delta} \in$ $B_{r}\left(x_{0}\right)$, for some $m \geq 0$. Then, using (b), (3.26) and Lemma 3.2, we have

$$
\begin{aligned}
\left\|x_{m+1, \alpha_{k}, s}^{\delta}-x_{0}\right\| & \leq\left\|x_{m+1, \alpha_{k}}^{\delta}-x_{m, \alpha_{k}, s}^{\delta}\right\|+\cdots+\left\|x_{1, \alpha_{k}, s}^{h,}-x_{0}\right\| \\
& \leq(q+1)\left(q^{2 m}+q^{2(m-1)}+\cdots+1\right) e_{0, \alpha_{k}, s}^{\delta} \\
& \leq(q+1) \frac{1-\left(q^{2 m+1}\right)}{1-q^{2}} e_{0, \alpha_{k}, s}^{\delta} \\
& \leq \frac{\gamma_{\rho}}{1-q}<r,
\end{aligned}
$$

i.e., $x_{m+1, \alpha_{k}, s}^{\delta} \in B_{r}\left(x_{0}\right)$ and

$$
\begin{aligned}
\left\|y_{m+1, \alpha_{k}, s}^{\delta}-x_{0}\right\| \leq & \left\|y_{m+1, \alpha_{k}, s}^{\delta}-x_{m+1, \alpha_{k}, s}^{\delta}\right\| \\
& +\left\|x_{m+1, \alpha_{k}, s}^{\delta}-x_{0}\right\| \\
\leq & \left(q^{2(m+1)}+\cdots+q^{3}+q^{2}+q+1\right) e_{0, \alpha_{k}, s}^{\delta} \\
\leq & \frac{\gamma_{\rho}}{1-q}<r,
\end{aligned}
$$


i.e., $y_{m+1, \alpha_{k}, s}^{\delta} \in B_{r}\left(x_{0}\right)$. Thus, by induction, $x_{n, \alpha_{k}, s}^{\delta}, y_{n, \alpha_{k}, s}^{\delta} \in B_{r}\left(x_{0}\right)$, for all $n \geq 0$. This completes the proof of Theorem 3.3.

Theorem 3.4. Let $x_{n, \alpha_{k}, s}^{\delta}$ and $y_{n, \alpha_{k}, s}^{\delta}$ be as in (1.7) and (1.8), respectively, with $\alpha=\alpha_{k}$ and $\delta \in\left(0, \delta_{0}\right]$, and let the assumptions of Theorem 3.3 hold. Then $\left(x_{n, \alpha_{k}, s}^{\delta}\right)$ is a Cauchy sequence in $B_{r}\left(x_{0}\right)$ and converges, say, to $x_{\alpha_{k}, s}^{\delta} \in \overline{B_{r}\left(x_{0}\right)}$. Further, $F\left(x_{\alpha_{k}, s}^{\delta}\right)=z_{\alpha_{k}, s}^{\delta}$ and

$$
\left\|x_{n, \alpha_{k}, s}^{\delta}-x_{\alpha_{k}, s}^{\delta}\right\| \leq C q^{2 n}
$$

where $C=\left(\gamma_{\rho}\right) /(1-q)$.

Proof. Using relations (b) and (c) of Theorem 3.3, we obtain

$$
\begin{aligned}
\left\|x_{n+m, \alpha_{k}, s}^{\delta}-x_{n, \alpha_{k}, s}^{\delta}\right\| \leq & \sum_{i=0}^{i=m-1}\left\|x_{n+i+1, \alpha_{k}, s}^{\delta}-x_{n+i, \alpha_{k}, s}^{\delta}\right\| \\
\leq & \sum_{i=0}^{i=m-1}(1+q) e_{n+i, \alpha_{k}, s}^{\delta} \\
\leq & \sum_{i=0}^{i=m-1}(1+q) q^{2(n+i)} e_{0, \alpha_{k}, s}^{\delta} \\
= & (1+q) q^{2 n} e_{0, \alpha_{k}, s}^{\delta}+(1+q) q^{2(n+1)} e_{0, \alpha_{k}, s}^{\delta} \\
& +\cdots+(1+q) q^{2(n+m)} e_{0, \alpha_{k}, s}^{\delta} \\
\leq & (1+q) q^{2 n}\left(1+q^{2}+q^{2(2)}+\cdots+q^{2 m}\right) e_{0, \alpha_{k}, s}^{\delta} \\
\leq & q^{2 n}\left[\frac{1-\left(q^{2}\right)^{m+1}}{1-q}\right] \gamma_{\rho} \leq C q^{2 n} .
\end{aligned}
$$

Thus, $x_{n, \alpha_{k}, s}^{\delta}$ is a Cauchy sequence in $B_{r}\left(x_{0}\right)$, and hence it converges, say, to $x_{\alpha_{k}, s}^{\delta} \in \overline{B_{r}\left(x_{0}\right)}$. Observe that

$$
\begin{aligned}
\left\|F\left(x_{n, \alpha_{k}, s}^{\delta}\right)-z_{\alpha_{k}, s}^{\delta}\right\| & =\left\|F^{\prime}\left(x_{0}\right)\left(x_{n, \alpha_{k}, s}^{\delta}-y_{n, \alpha_{k}, s}^{\delta}\right)\right\| \\
& \leq\left\|F^{\prime}\left(x_{0}\right)\right\|\left\|x_{n, \alpha_{k}, s}^{\delta}-y_{n, \alpha_{k}, s}^{\delta}\right\| \\
& \leq M e_{n, \alpha_{k}, s}^{\delta} \leq M q^{2 n} \gamma_{\rho} .
\end{aligned}
$$

Now, by letting $n \rightarrow \infty$ in (3.27) we obtain $F\left(x_{\alpha_{k}, s}^{\delta}\right)=z_{\alpha_{k}, s}^{\delta}$. This completes the proof. 
Hereafter, we assume that $\left\|\widehat{x}-x_{0}\right\|<\rho \leq r$.

Theorem 3.5. Suppose that Assumption 3.1 holds. Then

$$
\left\|\widehat{x}-x_{\alpha_{k}, s}^{\delta}\right\| \leq \frac{\beta}{1-q}\left\|F(\widehat{x})-z_{\alpha_{k}, s}^{\delta}\right\| .
$$

Proof. Note that $q<1$ and, by Assumption 3.1, we have

$$
\begin{aligned}
\left\|\widehat{x}-x_{\alpha_{k}, s}^{\delta}\right\| \leq & \| \widehat{x}-x_{\alpha_{k}, s}^{\delta}+F^{\prime}\left(x_{0}\right)^{-1} \\
& \times\left[F\left(x_{\alpha_{k}, s}^{\delta}\right)-F(\widehat{x})+F(\widehat{x})-z_{\alpha_{k}, s}^{\delta}\right] \| \\
\leq & \left\|F^{\prime}\left(x_{0}\right)^{-1}\left[F^{\prime}\left(x_{0}\right)\left(\widehat{x}-x_{\alpha_{k}, s}^{\delta}\right)+F\left(x_{\alpha_{k}, s}^{\delta}\right)-F(\widehat{x})\right]\right\| \\
& +\left\|F^{\prime}\left(x_{0}\right)^{-1}\left(F(\widehat{x})-z_{\alpha_{k}, s}^{\delta}\right)\right\| \\
\leq & k_{0}\left\|x_{0}-\widehat{x}-t\left(x_{\alpha_{k}, s}^{\delta}-\hat{x}\right)\right\|\left\|\widehat{x}-x_{\alpha_{k}, s}^{\delta}\right\|+\beta\left\|F(\widehat{x})-z_{\alpha_{k}, s}^{\delta}\right\| \\
\leq & k_{0} r\left\|\widehat{x}-x_{\alpha_{k}, s}^{\delta}\right\|+\beta\left\|F(\widehat{x})-z_{\alpha_{k}, s}^{\delta}\right\| .
\end{aligned}
$$

Hence, the theorem is proved.

Theorem 3.4 and Theorem 3.5 together imply the following theorem.

Theorem 3.6. Let $x_{n, \alpha_{k}, s}^{\delta}$ be as in (1.7) with $\alpha=\alpha_{k}$ and $\delta \in\left(0, \delta_{0}\right]$, and let the assumptions in Theorems 3.4 and 3.5 hold. Then

$$
\left\|\widehat{x}-x_{n, \alpha_{k}, s}^{\delta}\right\| \leq C q^{2 n}+\frac{\beta}{1-q}\left\|F(\hat{x})-z_{\alpha_{k}, s}^{\delta}\right\|,
$$

where $C$ is as in Theorem 3.4 .

Theorem 3.7. Let $x_{n, \alpha_{k}, s}^{\delta}$ be as in (1.7) with $\alpha=\alpha_{k}$ and $\delta \in\left(0, \delta_{0}\right]$, and let the assumptions in Theorems 2.5 and 3.6 hold. Let

$$
n_{k}:=\min \left\{n: q^{2 n} \leq \alpha_{k}^{-a / 2(s+a)} \delta\right\} .
$$

Then

$$
\left\|\widehat{x}-x_{n_{k}, \alpha_{k}, s}^{\delta}\right\|=O\left(\psi_{s, a}^{-1}(\delta)\right) .
$$




\section{2 .}

Case II: $F^{\prime}\left(x_{0}\right)$ is non-invertible and $F$ is a monotone operator. In this section, let $X$ be a real Hilbert space. We consider the two step iterative method defined as in (1.11) and (1.12) with $\alpha_{k}$ in place of $\alpha$ for approximating the zero $\widetilde{x}_{\alpha_{k}, s}^{\delta}$ of the equation,

$$
F(x)+\frac{\alpha_{k}}{c} L^{s / 2}\left(x-x_{0}\right)=z_{\alpha_{k}, s}^{\delta},
$$

and then we show that $\widetilde{x}_{\alpha_{k}, s}^{\delta}$ is an approximation to the solution $\widehat{x}$ of (1.1).

Let $F^{\prime}\left(x_{0}\right) \in L(X)$ be a bounded positive self-adjoint operator on $X$ and $B_{s}:=L^{-s / 4} F^{\prime}\left(x_{0}\right) L^{-s / 4}$. Usually, for the analysis of regularization methods in Hilbert scales, an assumption of the form (cf. $[\mathbf{1}, \mathbf{1 3}]$ )

$$
\left\|F^{\prime}(\widehat{x}) x\right\| \sim\|x\|_{-b}, \quad x \in X,
$$

on the degree of ill-posedness is used. In this paper, instead of (3.29), we require only a weaker assumption:

$$
d_{1}\|x\|_{-b} \leq\left\|F^{\prime}\left(x_{0}\right) x\right\| \leq d_{2}\|x\|_{-b}, \quad x \in D(F),
$$

for some reals $b, d_{1}$ and $d_{2}$.

Note that (3.30) is simpler than (3.29). Now we define $f_{1}$ and $g_{1}$ by

$$
f_{1}(t)=\min \left\{d_{1}^{t}, d_{2}^{t}\right\}, \quad g_{1}(t)=\max \left\{d_{1}^{t}, d_{2}^{t}\right\}, \quad t \in \mathbf{R},|t| \leq 1 .
$$

One of the crucial steps for proving the results in this paper is the following proposition.

Proposition 3.8 (see [7], Proposition 3.1). For $s>0$ and $|\nu| \leq 1$, $f_{1}(\nu / 2)\|x\|_{(-\nu(s+b)) / 2} \leq\left\|B_{s}^{\nu / 2} x\right\| \leq g_{1}(\nu / 2)\|x\|_{(-\nu(s+b)) / 2}, \quad x \in H$.

Let

$$
\psi_{2}(s):=\frac{g_{1}(-s /(2(s+b)))}{f_{1}(s /(2(s+b)))}, \quad \overline{\psi_{2}(s)}:=\frac{g_{1}(s /(2(s+b)))}{f_{1}(s /(2(s+b)))},
$$


and let

$$
\widetilde{e}_{n, \alpha_{k}}^{\delta}:=\left\|\widetilde{y}_{n, \alpha_{k}}^{\delta}-\widetilde{x}_{n, \alpha_{k}}^{\delta}\right\|, \quad \text { for all } n \geq 0 .
$$

Let $\delta_{0}<1 /\left[4 k_{0} \psi(s) \psi_{2}(s) \overline{\psi_{2}(s)}\right] \alpha_{0}^{a /[2(s+a)]}$ and $\left\|\widehat{x}-x_{0}\right\| \leq \rho$, with

$$
\rho<\frac{1}{M \psi_{1}(s)}\left[\frac{1}{4 k_{0} \psi_{2}(s) \overline{\psi_{2}(s)}}-\psi(s) \alpha_{0}^{-a /[2(s+a)]} \delta_{0}\right]
$$

and

$$
\widetilde{\gamma}_{\rho}:=\psi_{2}(s)\left[\psi_{1}(s) M \rho+\psi(s) \alpha_{0}^{-a /[2(s+a)]} \delta_{0}\right] .
$$

Further, let

$$
\widetilde{r}_{1}=\frac{1-\sqrt{1-4 k_{0} \overline{\psi_{2}(s)}} \widetilde{\gamma}_{\rho}}{2 \overline{\psi_{2}(s)} k_{0}}
$$

and

$$
\widetilde{r}_{2}=\min \left\{\frac{1}{k_{0}}, \frac{1+\sqrt{1-4 k_{0} \overline{\psi_{2}(s)} \widetilde{\gamma}_{\rho}}}{2 \overline{\psi_{2}(s)} k_{0}}\right\}
$$

For $\widetilde{r} \in\left(\widetilde{r}_{1}, \widetilde{r}_{2}\right)$, let

$$
\widetilde{q}=\overline{\psi_{2}(s)} k_{0} \widetilde{r}
$$

Then $\widetilde{q}<1$.

Lemma 3.9. Let $\widetilde{e}_{0, \alpha_{k}, s}^{\delta}$ be as in (3.31), and let Proposition 3.8 hold. Then $\widetilde{e}_{0, \alpha_{k}, s}^{\delta}<\widetilde{\gamma}_{\rho}$.

Proof. Observe that

$$
\begin{aligned}
\widetilde{e}_{0, \alpha_{k}, s}^{\delta} & =\left\|\widetilde{y}_{0, \alpha_{k}, s}^{\delta}-\widetilde{x}_{0, \alpha_{k}, s}^{\delta}\right\| \\
& =\left\|\left(F^{\prime}\left(x_{0}\right)+\frac{\alpha_{k}}{c} L^{s / 2}\right)^{-1}\left(F\left(x_{0}\right)-z_{\alpha_{k}, s}^{\delta}\right)\right\|
\end{aligned}
$$




$$
\begin{aligned}
& \leq \| L^{-s / 4}\left(L^{-s / 4} F^{\prime}\left(x_{0}\right) L^{-s / 4}+\frac{\alpha_{k}}{c} I\right)^{-1} L^{-s / 4} \\
& \quad \times\left(F\left(x_{0}\right)-z_{\alpha_{k}, s}^{\delta}\right) \| \\
& \leq \frac{1}{f_{1}(s /(2(s+b)))} \| B_{s}^{s /(2(s+b))}\left(B_{s}+\frac{\alpha_{k}}{c} I\right)^{-1} L^{-s / 4} \\
& \quad \times\left(F\left(x_{0}\right)-z_{\alpha_{k}, s}^{\delta}\right) \| \\
& \leq \frac{1}{f_{1}(s /(2(s+b)))} \|\left(B_{s}+\frac{\alpha_{k}}{c} I\right)^{-1} B_{s}^{s /(s+b)} B_{s}^{-s /(2(s+b))} \\
& \leq \frac{g_{1}(-s /(2(s+b)))}{f_{1}(s /(2(s+b)))}\left(\frac{\alpha_{k}}{c}\right)^{-b /(s+b)}\left\|F\left(x_{0}\right)-z_{\alpha_{k}, s}^{\delta}\right\| \\
& \leq \psi_{2}(s)\left[\left\|F\left(x_{0}\right)-z_{\alpha_{k}, s}\right\|+\left\|z_{\alpha_{k}, s}-z_{\alpha_{k}, s}^{\delta}\right\|\right] .
\end{aligned}
$$

Now, using (2.14) and (2.16) in (3.33), one can see that

$$
\begin{aligned}
\widetilde{e}_{0, \alpha_{k}, s}^{\delta} & \leq \psi_{2}(s)\left[\psi_{1}(s)\left\|F(\widehat{x})-F\left(x_{0}\right)\right\|+\psi(s) \alpha^{-a /(2(s+a))} \delta\right] \\
& \leq \psi_{2}(s)\left[\psi_{1}(s) M \rho+\psi(s) \alpha_{0}^{-a / 2(s+a)} \delta_{0}\right]=\widetilde{\gamma_{\rho}}
\end{aligned}
$$

Lemma 3.10. Let Proposition 3.8 hold. Then, for all $h \in X$,

$$
\left\|\left(F^{\prime}\left(x_{0}\right)+\frac{\alpha_{k}}{c} L^{s / 2}\right)^{-1} F^{\prime}\left(x_{0}\right) h\right\| \leq \overline{\psi_{2}(s)}\|h\| .
$$

Proof. Observe that, by Proposition 3.8,

$$
\begin{aligned}
\left\|\left(F^{\prime}\left(x_{0}\right)+\frac{\alpha_{k}}{c} L^{s / 2}\right)^{-1} F^{\prime}\left(x_{0}\right) h\right\| & \\
=\| L^{-s / 4}\left(L^{-s / 4} F^{\prime}\left(x_{0}\right) L^{-s / 4}+\right. & \left.\frac{\alpha_{k}}{c} I\right)^{-1} L^{-s / 4} \\
& \times F^{\prime}\left(x_{0}\right) L^{-s / 4} L^{s / 4} h \|
\end{aligned}
$$




$$
\begin{aligned}
& \leq \frac{1}{f_{1}(s /(2(s+b)))}\left\|B_{s}^{s /(2(s+b))}\left(B_{s}+\frac{\alpha_{k}}{c} I\right)^{-1} B_{s} L^{s / 4} h\right\| \\
& \leq \frac{1}{f_{1}(s /(2(s+b)))}\left\|\left(B_{s}+\frac{\alpha_{k}}{c} I\right)^{-1} B_{s}\right\|\left\|B_{s}^{s /(2(s+b))} L^{s / 4} h\right\| \\
& \leq \frac{g_{1}(s /(2(s+b)))}{f_{1}(s /(2(s+b)))}\left\|L^{s / 4} h\right\|_{-s / 2} \\
& \leq \frac{g_{1}(s /(2(s+b)))}{f_{1}(s /(2(s+b)))}\|h\| .
\end{aligned}
$$

This completes the proof of Lemma 3.10.

Theorem 3.11. Let $\widetilde{e}_{n, \alpha_{k}, s}^{\delta}$ and $\widetilde{q}$ be as in equations (3.31) and (3.32), respectively, $\widetilde{y}_{n, \alpha_{k}, s}^{\delta}$ and $\widetilde{x}_{n, \alpha_{k}, s}^{\delta}$ as defined in (1.11) and (1.12), respectively, with $\alpha=\alpha_{k}$ and $\delta \in\left(0, \delta_{0}\right]$. Then, under Assumption 3.1 and Lemma 3.9, $\widetilde{x}_{n, \alpha_{k}, s}^{\delta}, \widetilde{y}_{n, \alpha_{k}, s}^{\delta} \in B_{\tilde{r}}\left(x_{0}\right)$, and the following estimates hold for all $n \geq 0$ :

(a) $\left\|\widetilde{x}_{n, \alpha_{k}, s}^{\delta}-\widetilde{y}_{n-1, \alpha_{k}, s}^{\delta}\right\| \leq \widetilde{q}\left\|\widetilde{y}_{n-1, \alpha_{k}, s}^{\delta}-\widetilde{x}_{n-1, \alpha_{k}, s}^{\delta}\right\|$;

(b) $\left\|\widetilde{x}_{n, \alpha_{k}, s}^{\delta}-\widetilde{x}_{n-1, \alpha_{k}, s}^{\delta}\right\| \leq(1+\widetilde{q})\left\|\widetilde{y}_{n-1, \alpha_{k}, s}^{\delta}-\widetilde{x}_{n-1, \alpha_{k}, s}^{\delta}\right\|$;

(c) $\left\|\widetilde{y}_{n, \alpha_{k}, s}^{\delta}-\widetilde{x}_{n, \alpha_{k}, s}^{\delta}\right\| \leq \widetilde{q}^{2}\left\|\widetilde{y}_{n-1, \alpha_{k}, s}^{\delta}-\widetilde{x}_{n-1, \alpha_{k}, s}^{\delta}\right\|$;

(d) $\widetilde{e}_{n, \alpha_{k}, s}^{\delta} \leq \widetilde{q}^{2 n} \widetilde{\gamma}_{\rho}$, for all $n \geq 0$.

Proof. If $\widetilde{x}_{n, \alpha_{k}, s}^{\delta}, y_{n, \alpha_{k}, s}^{\delta} \in B_{\tilde{r}}\left(x_{0}\right)$, then by Assumption 3.1,

$$
\begin{aligned}
& \widetilde{x}_{n+1, \alpha_{k}, s}^{\delta}-\widetilde{y}_{n, \alpha_{k}, s}^{\delta}=\left(F^{\prime}\left(x_{0}\right)+\frac{\alpha_{k}}{c} L^{s / 2}\right)^{-1} \\
& \times\left[F^{\prime}\left(x_{0}\right)\left(\widetilde{y}_{n, \alpha_{k}, s}^{\delta}-\widetilde{x}_{n, \alpha_{k}, s}^{\delta}\right)\right. \\
&\left.\quad-\left(F\left(\widetilde{y}_{n, \alpha_{k}, s}^{\delta}\right)-F\left(\widetilde{x}_{n, \alpha_{k}, s}^{\delta}\right)\right)\right] \\
&=\left(F^{\prime}\left(x_{0}\right)+\frac{\alpha_{k}}{c} L^{s / 2}\right)^{-1} \int_{0}^{1}\left[F^{\prime}\left(x_{0}\right)-F^{\prime}\left(\widetilde{x}_{n, \alpha_{k}, s}^{\delta}\right.\right. \\
&\left.+t\left(\widetilde{y}_{n, \alpha_{k}, s}^{\delta}-\widetilde{x}_{n, \alpha_{k}, s}^{\delta}\right)\right]\left(\widetilde{y}_{n, \alpha_{k}, s}^{\delta}-\widetilde{x}_{n, \alpha_{k}, s}^{\delta}\right) d t \\
&=\left(F^{\prime}\left(x_{0}\right)+\frac{\alpha_{k}}{c} L^{s / 2}\right)^{-1} F^{\prime}\left(x_{0}\right) \int_{0}^{1} \Phi\left(x_{0}, \widetilde{x}_{n, \alpha_{k}, s}^{\delta}\right. \\
&\left.+t\left(\widetilde{y}_{n, \alpha_{k}, s}^{\delta}-\widetilde{x}_{n, \alpha_{k}, s}^{\delta}\right), \widetilde{y}_{n, \alpha_{k}}^{\delta}-\widetilde{x}_{n, \alpha_{k}, s}^{\delta}\right) d t,
\end{aligned}
$$


and hence, by Lemma 3.10 and Assumption 3.1, we have

$$
\begin{aligned}
\left\|\widetilde{x}_{n+1, \alpha_{k}, s}^{\delta}-\widetilde{y}_{n, \alpha_{k}, s}^{\delta}\right\| & \leq \overline{\psi_{2}(s)} \| \int_{0}^{1} \Phi\left(x_{0}, \widetilde{x}_{n, \alpha_{k}, s}^{\delta}\right. \\
& \left.\quad+t\left(\widetilde{y}_{n, \alpha_{k}, s}^{\delta}-\widetilde{x}_{n, \alpha_{k}, s}^{\delta}\right), \widetilde{y}_{n, \alpha_{k}, s}^{\delta}-\widetilde{x}_{n, \alpha_{k}, s}^{\delta}\right) d t \| \\
& \leq \overline{\psi_{2}(s)} k_{0} r\left\|\widetilde{y}_{n, \alpha_{k}, s}^{\delta}-\widetilde{x}_{n, \alpha_{k}, s}^{\delta}\right\| .
\end{aligned}
$$

This proves (a).

Now (b) follows from (a) and the triangle inequality:

$$
\begin{aligned}
\left\|\widetilde{x}_{n, \alpha_{k}, s}^{\delta}-\widetilde{x}_{n-1, \alpha_{k}, s}^{\delta}\right\| \leq & \left\|\widetilde{x}_{n, \alpha_{k}, s}^{\delta}-\widetilde{y}_{n-1, \alpha_{k}, s}^{\delta}\right\| \\
& +\left\|\widetilde{y}_{n-1, \alpha_{k}, s}^{\delta}-\widetilde{x}_{n-1, \alpha_{k}, s}^{\delta}\right\| .
\end{aligned}
$$

Again, (c) follows from (a), Assumption 3.1, Lemma 3.10 and the following expression

$$
\begin{aligned}
\widetilde{e}_{n, \alpha_{k}, s}^{\delta}= & \|\left(F^{\prime}\left(x_{0}\right)+\frac{\alpha_{k}}{c} L^{s / 2}\right)^{-1}\left[F^{\prime}\left(x_{0}\right)\left(\widetilde{x}_{n, \alpha_{k}, s}^{\delta}-\widetilde{y}_{n-1, \alpha_{k}, s}^{\delta}\right)\right. \\
= & \left.\|\left(F\left(\widetilde{x}_{n, \alpha_{k}, s}^{\delta}\right)-F\left(\widetilde{y}_{n-1, \alpha_{k}, s}^{\delta}\right)\right)\right] \| \\
& \left.\times \int_{0}^{1}\left[x_{0}\right)+\frac{\alpha_{k}}{c} L^{s / 2}\right)^{-1}\left(x_{0}\right)-\left(F^{\prime}\left(\widetilde{y}_{n-1, \alpha_{k}, s}^{\delta}\right)\right. \\
& \left.\left.+t\left(\widetilde{y}_{n-1, \alpha_{k}, s}^{\delta}-\widetilde{x}_{n, \alpha_{k}, s}^{\delta}\right)\right)\right]\left(\widetilde{x}_{n, \alpha_{k}, s}^{\delta}-\widetilde{y}_{n-1, \alpha_{k}, s}^{\delta}\right) d t \| .
\end{aligned}
$$

Further, (d) follows from (c).

The remaining part of the proof is analogous to the proof of Theorem 3.3.

Next, we go to the main result of this section.

Theorem 3.12. Let $\widetilde{y}_{n, \alpha_{k}, s}^{\delta}$ and $\widetilde{x}_{n, \alpha_{k}, s}^{\delta}$ be as in (1.11) and (1.12), respectively, with $\alpha=\alpha_{k}, \delta \in\left(0, \delta_{0}\right]$ and the assumptions of Theorem 3.11 holding. Then $\left(\widetilde{x}_{n, \alpha_{k}, s}^{\delta}\right)$ is a Cauchy sequence in $B_{\tilde{r}}\left(x_{0}\right)$ 
and converges, say, to $\widetilde{x}_{\alpha_{k}, s}^{\delta} \in \overline{B_{\tilde{r}}\left(x_{0}\right)}$. Further, $F\left(\widetilde{x}_{\alpha_{k}, s}^{\delta}\right)-z_{\alpha_{k}, s}^{\delta}+$ $\left(\alpha_{k} / c\right) L^{s / 2}\left(\widetilde{x}_{\alpha_{k}, s}^{\delta}-x_{0}\right)=0$ and $\left\|\widetilde{x}_{n, \alpha_{k}, s}^{\delta}-\widetilde{x}_{\alpha_{k}, s}^{\delta}\right\| \leq \widetilde{C} \widetilde{q}^{2 n}$, where $\widetilde{C}=\left(\widetilde{\gamma}_{\rho}\right) /(1-\widetilde{q})$.

Proof. Analogous to the proof of Theorem 3.4, one can see that $\left(\widetilde{x}_{n, \alpha_{k}, s}^{\delta}\right)$ is a Cauchy sequence in $B_{\widetilde{r}}\left(x_{0}\right)$, and hence it converges, say, to $\widetilde{x}_{\alpha_{k}, s}^{\delta} \in \overline{B_{\widetilde{r}}\left(x_{0}\right)}$. Observe that, from (3.11),

$$
\begin{aligned}
\| F\left(\widetilde{x}_{n, \alpha_{k}, s}^{\delta}\right)- & z_{\alpha_{k}, s}^{\delta}+\frac{\alpha_{k}}{c} L^{s / 2}\left(\widetilde{x}_{n, \alpha_{k}, s}^{\delta}-x_{0}\right) \| \\
& =\left\|\left(F^{\prime}\left(x_{0}\right)+\frac{\alpha_{k}}{c} L^{s / 2}\right)\left(\widetilde{y}_{n, \alpha_{k}, s}^{\delta}-\widetilde{x}_{n, \alpha_{k}, s}^{\delta}\right)\right\| \\
& \leq\left\|F^{\prime}\left(x_{0}\right)+\frac{\alpha_{k}}{c} L^{s / 2}\right\|_{X_{s} \rightarrow X}\left\|\widetilde{y}_{n, \alpha_{k}, s}^{\delta}-\widetilde{x}_{n, \alpha_{k}, s}^{\delta}\right\| \\
& \leq\left\|F^{\prime}\left(x_{0}\right)+\frac{\alpha_{k}}{c} L^{s / 2}\right\|_{X_{s} \rightarrow X} \widetilde{e}_{n, \alpha_{k}, s}^{\delta} \\
& \leq\left\|F^{\prime}\left(x_{0}\right)+\frac{\alpha_{k}}{c} L^{s / 2}\right\|_{X_{s} \rightarrow X} \widetilde{q}^{2 n} \widetilde{\gamma}_{\rho} .
\end{aligned}
$$

Now, by letting $n \rightarrow \infty$ in (3.34), we obtain $F\left(\widetilde{x}_{\alpha_{k}, s}^{\delta}\right)+\left(\alpha_{k} / c\right) L^{s / 2}\left(\widetilde{x}_{\alpha_{k}, s}^{\delta}\right.$ $\left.-x_{0}\right)=z_{\alpha_{k}, s}^{\delta}$. This completes the proof.

In addition to Assumption 2.2, we use the following assumption to obtain the error estimate for $\left\|\widehat{x}-\widetilde{x}_{\alpha_{k}, s}^{\delta}\right\|$.

Assumption 3.13. There exists a continuous, strictly monotonically increasing function $\varphi_{1}:\left(0,\left\|B_{s}\right\|\right] \rightarrow(0, \infty)$ such that the following conditions hold:

- $\lim _{\lambda \rightarrow 0} \varphi_{1}(\lambda)=0$,

$$
\sup _{\lambda>0} \frac{\alpha \varphi_{1}(\lambda)}{\lambda+\alpha} \leq \varphi_{1}(\alpha) \text { for all } \lambda \in\left(0,\left\|B_{s}\right\|\right],
$$

- there exists $w \in X$ with $\|w\| \leq E_{2}$, such that

$$
B_{s}^{s /(2(s+b))} L^{s / 4}\left(x_{0}-\hat{x}\right)=\varphi_{1}\left(B_{s}\right) w .
$$


- For each $x \in B_{\tilde{r}}\left(x_{0}\right)$, there exists a bounded linear operator $G\left(x, x_{0}\right)$ (cf. [19]) such that

$$
F^{\prime}(x)=F^{\prime}\left(x_{0}\right) G\left(x, x_{0}\right)
$$

with $\left\|G\left(x, x_{0}\right)\right\| \leq k_{1}$.

Remark 3.14. If $x_{0}-\widehat{x} \in X_{t_{1}}$, i.e., $\left\|x_{0}-\widehat{x}\right\|_{t_{1}} \leq E_{1}$ for some positive constant $E_{1}$ and $0 \leq t_{1} \leq s+b$, then, as in Remark 2.3, we have $B_{s}^{s /(2(s+b))} L^{s / 4}\left(x_{0}-\widehat{x}\right)=\varphi_{1}\left(B_{s}\right) w$ where $\varphi_{1}(\lambda)=\lambda^{t_{1} /(s+b)}, w=$ $B_{s}^{\left(s-2 t_{1}\right) /(2(s+b))} L^{s / 4}\left(\widehat{x}-x_{0}\right)$ and $\|w\| \leq g_{1}\left(\left(s-2 t_{1}\right) /(2(s+b))\right) E_{1}=$ : $E_{2}$.

Assume that $k_{1}<(1 /(1-c))\left[\left(1 / \overline{\psi_{2}(s)}\right)-k_{0} \widetilde{r}\right]$ and, for the sake of simplicity, assume that $\varphi_{1}(\alpha) \leq \varphi(\alpha)$ for $\alpha>0$. Let $\psi_{3}(s):=$ $E_{2} /\left[f_{1}(s /(2(s+b)))\right]$.

Theorem 3.15. Suppose $\widetilde{x}_{\alpha_{k}, s}^{\delta}$ is the solution of (3.28) and Assumptions 3.1 and 3.13 hold. Then

$$
\left\|\widehat{x}-\widetilde{x}_{\alpha_{k}, s}^{\delta}\right\|=O\left(\psi_{s, a}^{-1}(\delta)\right) .
$$

Proof. Note that $c\left(F\left(\widetilde{x}_{\alpha_{k}, s}^{\delta}\right)-z_{\alpha_{k}, s}^{\delta}\right)+\alpha_{k} L^{s / 2}\left(\widetilde{x}_{\alpha_{k}, s}^{\delta}-x_{0}\right)=0$, so

$$
\begin{aligned}
\left(F^{\prime}\left(x_{0}\right)+\alpha_{k} L^{s / 2}\right)\left(\widetilde{x}_{\alpha_{k}, s}^{\delta}-\widehat{x}\right)= & \left(F^{\prime}\left(x_{0}\right)+\alpha_{k} L^{s / 2}\right)\left(\widetilde{x}_{\alpha_{k}, s}^{\delta}-\widehat{x}\right) \\
& -c\left(F\left(\widetilde{x}_{\alpha_{k}, s}^{\delta}\right)-z_{\alpha_{k}, s}^{\delta}\right) \\
& -\alpha_{k} L^{s / 2}\left(\widetilde{x}_{\alpha, s}^{\delta}-x_{0}\right) \\
= & \alpha_{k} L^{s / 2}\left(x_{0}-\widehat{x}\right)+F^{\prime}\left(x_{0}\right)\left(\widetilde{x}_{\alpha_{k}, s}^{\delta}-\widehat{x}\right) \\
& -c\left[F\left(\widetilde{x}_{\alpha_{k}, s}^{\delta}\right)-z_{\alpha_{k}, s}^{\delta}\right] \\
= & \alpha_{k} L^{s / 2}\left(x_{0}-\widehat{x}\right)+F^{\prime}\left(x_{0}\right)\left(\widetilde{x}_{\alpha_{k}, s}^{\delta}-\widehat{x}\right) \\
& -c\left[F\left(\widetilde{x}_{\alpha_{k}, s}^{\delta}\right)-F(\widehat{x})+F(\widehat{x})-z_{\alpha_{k}, s}^{\delta}\right] \\
= & \alpha_{k} L^{s / 2}\left(x_{0}-\widehat{x}\right)-c\left(F(\widehat{x})-z_{\alpha_{k}, s}^{\delta}\right) \\
& +F^{\prime}\left(x_{0}\right)\left(x_{\alpha_{k}, s}^{\delta}-\widehat{x}\right)-c\left[F\left(\widetilde{x}_{\alpha_{k}, s}^{\delta}\right)-F(\widehat{x})\right] .
\end{aligned}
$$


Thus, since $0<c<\alpha_{k}$, we have

$$
\begin{aligned}
\left\|\widetilde{x}_{\alpha_{k}, s}^{\delta}-\widehat{x}\right\| \leq & \left\|\alpha_{k}\left(F^{\prime}\left(x_{0}\right)+\alpha_{k} L^{s / 2}\right)^{-1} L^{s / 2}\left(x_{0}-\widehat{x}\right)\right\| \\
& +\|\left(F^{\prime}\left(x_{0}\right)+\alpha_{k} L^{s / 2}\right)^{-1} \\
& \times c\left(F(\widehat{x})-z_{\alpha_{k}, s}^{\delta}\right)\|+\|\left(F^{\prime}\left(x_{0}\right)+\alpha_{k} L^{s / 2}\right)^{-1} \\
& \times\left[F^{\prime}\left(x_{0}\right)\left(\widetilde{x}_{\alpha_{k}, s}^{\delta}-\widehat{x}\right)-c\left(F\left(\widetilde{x}_{\alpha_{k}, s}^{\delta}\right)-F(\widehat{x})\right)\right] \| \\
\leq & \Gamma_{1}+\overline{\psi^{2}(s)}\left\|F(\widehat{x})-z_{\alpha_{k}, s}^{\delta}\right\|+\Gamma_{2},
\end{aligned}
$$

where

$$
\begin{aligned}
\Gamma_{1}:= & \left\|\alpha_{k}\left(F^{\prime}\left(x_{0}\right)+\alpha_{k} L^{s / 2}\right)^{-1} L^{s / 2}\left(x_{0}-\widehat{x}\right)\right\|, \\
\Gamma_{2}:= & \|\left(F^{\prime}\left(x_{0}\right)+\alpha_{k} L^{s / 2}\right)^{-1}\left[F^{\prime}\left(x_{0}\right)\left(\widetilde{x}_{\alpha_{k}, s}^{\delta}-\widehat{x}\right)\right. \\
& \left.-c\left(F\left(\widetilde{x}_{\alpha_{k}, s}^{\delta}\right)-F(\widehat{x})\right)\right] \| .
\end{aligned}
$$

Note that, by Assumption 3.13,

(3.36)

$$
\begin{aligned}
\Gamma_{1} & \leq\left\|\alpha_{k} L^{-s / 4}\left(B_{s}+\alpha_{k} I\right)^{-1} L^{s / 4}\left(x_{0}-\hat{x}\right)\right\| \\
& \leq \frac{1}{f_{1}((s /(2(s+b))))}\left\|\alpha_{k}\left(B_{s}+\alpha_{k} I\right)^{-1} B_{s}^{s /((2(s+b)))} L^{s / 4}\left(x_{0}-\widehat{x}\right)\right\| \\
& \leq \frac{1}{f_{1}((s / 2(s+b)))} \varphi_{1}\left(\alpha_{k}\right) E_{2}
\end{aligned}
$$

and

$$
\begin{aligned}
\Gamma_{2}= & \|\left(F^{\prime}\left(x_{0}\right)+\alpha_{k} L^{s / 2}\right)^{-1} \\
& \times \int_{0}^{1}\left[F^{\prime}\left(x_{0}\right)-c F^{\prime}\left(\widehat{x}+t\left(\widetilde{x}_{\alpha_{k}, s}^{\delta}-\widehat{x}\right)\right)\right]\left(\widetilde{x}_{\alpha_{k}, s}^{\delta}-\widehat{x}\right) d t \| \\
\leq & \|\left(F^{\prime}\left(x_{0}\right)+\alpha_{k} L^{s / 2}\right)^{-1} \\
& \times \int_{0}^{1}\left[F^{\prime}\left(x_{0}\right)-F^{\prime}\left(\widehat{x}+t\left(\widetilde{x}_{\alpha_{k}, s}^{\delta}-\widehat{x}\right)\right)\right]\left(\widetilde{x}_{\alpha_{k}, s}^{\delta}-\widehat{x}\right) d t \| \\
& +(1-c) \|\left(F^{\prime}\left(x_{0}\right)+\alpha_{k} L^{s / 2}\right)^{-1} F^{\prime}\left(x_{0}\right)
\end{aligned}
$$




$$
\begin{aligned}
& \times \int_{0}^{1} G\left(\widehat{x}+t\left(\widetilde{x}_{\alpha_{k}, s}^{\delta}-\widehat{x}\right), x_{0}\right)\left(\widetilde{x}_{\alpha_{k}, s}^{\delta}-\widehat{x}\right) d t \| \\
\leq & \overline{\psi_{2}(s)} k_{0} \widetilde{r}\left\|\widetilde{x}_{\alpha_{k}, s}^{\delta}-\widehat{x}\right\|+\overline{\psi_{2}(s)}(1-c) k_{1}\left\|\widetilde{x}_{\alpha_{k}, s}^{\delta}-\widehat{x}\right\| .
\end{aligned}
$$

The last step follows from Lemma 3.10, Proposition 3.8, Assumptions 3.1 and 3.13. Hence, by (3.35)-(3.37), we have

$$
\begin{aligned}
\left\|\widetilde{x}_{\alpha_{k}, s}^{\delta}-\widehat{x}\right\| & \leq \frac{\psi_{3}(s) \varphi_{1}\left(\alpha_{k}\right)+\overline{\psi^{2}(s)}\left\|F(\widehat{x})-z_{\alpha_{k}, s}^{\delta}\right\|}{1-\left[(1-c) k_{1}+k_{0} \widetilde{r}\right] \overline{\psi_{2}(s)}} \\
& \leq \frac{\psi_{3}(s) \varphi_{1}\left(\alpha_{k}\right)+\overline{\psi^{2}(s)} C_{s}(2+(4 \eta) /(\eta-1)) \eta\left(\psi_{s, a}^{-1}(\delta)\right)}{1-\left[(1-c) k_{1}-k_{0} \widetilde{r}\right] \overline{\psi_{2}(s)}} \\
& =O\left(\psi_{s, a}^{-1}(\delta)\right) .
\end{aligned}
$$

This completes the proof of Theorem 3.15.

The following Theorem is a consequence of Theorems 3.12 and 3.15 .

Theorem 3.16. Let $\widetilde{x}_{n, \alpha_{k}, s}^{\delta}$ be as in (1.12) with $\alpha=\alpha_{k}$ and $\delta \in\left(0, \delta_{0}\right]$, and let the assumptions in Theorems 3.12 and 3.15 hold. Then

$$
\left\|\widehat{x}-\widetilde{x}_{n, \alpha_{k}, s}^{\delta}\right\| \leq \widetilde{C} q^{2 n}+O\left(\psi_{s, a}^{-1}(\delta)\right)
$$

where $\widetilde{C}$ is as in Theorem 3.12 .

Theorem 3.17. Let $\widetilde{x}_{n, \alpha_{k}, s}^{\delta}$ be as in (1.12) with $\alpha=\alpha_{k}$ and $\delta \in\left(0, \delta_{0}\right]$, and let the assumptions in Theorem 3.16 hold. Let

$$
n_{k}:=\min \left\{n: \widetilde{q}^{2 n} \leq \alpha_{k}^{-a /(2(s+a))} \delta\right\} .
$$

Then

$$
\left\|\widehat{x}-\widetilde{x}_{n_{k}, \alpha_{k}, s}^{\delta}\right\|=O\left(\psi_{s, a}^{-1}(\delta)\right) .
$$

4. Conclusion. In this paper we present a two step iterative regularization method for obtaining an approximate solution of an illposed Hammerstein type operator equation $K F(x)=y$ in the Hilbert 
scale setting where $K$ is a bounded linear operator and $F$ is a nonlinear operator. It is assumed that the available data is $y^{\delta}$ in place of the exact data $y$. Two cases of $F$ are discussed: (a) $F^{\prime}\left(x_{0}\right)^{-1}$ exists and is bounded and (b) $F$ is monotone and $F^{\prime}\left(x_{0}\right)$ is non-invertible. We considered the Hilbert space $\left(X_{t}\right)_{t \in \mathbf{R}}$ generated by $L$ for the analysis where $L: D(L) \rightarrow X$ is a linear, unbounded, self-adjoint, densely defined and strictly positive operator on $X$. In order to choose the regularization parameter $\alpha$, we used the adaptive scheme of Pereverzev and Schock [14].

Acknowledgments. Ms. Shobha thanks the National Institute of Technology Karnataka, India, for financial support.

\section{REFERENCES}

1. H. Egger and A. Neubauer, Preconditioning Landweber iteration in Hilbert scales, Numer. Math. 101 (2005), 643-662.

2. F. Faraci, Existence and multiplicity results for a nonlinear Hammerstein integral equation, Variat. Anal. Appl. 79 (2005), 359-371.

3. S. George, Newton-Tikhonov regularization of ill-posed Hammerstein operator equation, J. Inv. Ill-Posed Prob. 2 (2006), 135-146.

4. - Newton-Lavrentiev regularization of ill-posed Hammerstein operator equation, J. Inv. Ill-Posed Prob. 6 (2006), 573-582.

5. S. George and M. Kunhanandan, An iterative regularization method for Illposed Hammerstein type operator equation, J. Inv. Ill-Posed Prob. 17 (2009), 831-844.

6. S. George and M.T. Nair, A modified Newton-Lavrentiev regularization for nonlinear ill-posed Hammerstein-type operator equations, J. Complexity 24 (2008), $228-240$.

7. - Error bounds and parameter choice strategies for simplified regularization in Hilbert scales, Int. Equat. Oper. Theor. 29 (1997), 231-242.

8. S. George and M.E. Shobha, A regularized dynamical system method for nonlinear ill-posed Hammerstein type operator equations, J. Appl. Math. Bio. 1 (2011), 65-78.

9. M.A. Krasnoselskii, P.P. Zabreiko, E.I. Pustylnik and P.E. Sobolevskii, Integral operators in spaces of summable functions, Noordhoff International Publishing, Leyden, 1976.

10. S. Lu, S.V. Pereverzev, Y. Shao and U. Tautenhahn, On the generalized discrepancy principle for Tikhonov regularization in Hilbert scales, J. Integ. Equat. Appl. 22 (2010), 483-517.

11. P. Mathe and U. Tautenhahn, Error bounds for regularization methods in Hilbert scales by using operator monotonicity, Far East J. Math. Sci. 24 (2007), $1-21$. 
12. F. Natterer, Error bounds for Tikhonov regularization in Hilbert scales, Appl. Anal. 18 (1984), 29-37.

13. A. Neubauer, On Landweber iteration for non-linear ill-posed problems in Hilbert scales, Numer. Math. 85 (2000), 309-328.

14. S. Pereverzev and E. Schock, On the adaptive selection of the parameter in regularization of ill-posed problems, SIAM. J. Numer. Anal. 43 (2005), 2060-2076.

15. Jin Qi-nian, Error estimates of some Newton-type methods for solving nonlinear inverse problems in Hilbert scales, Inv. Prob. 16 (2000), 187-197.

16. Jin Qinian and U. Tautenhahn, Inexact Newton regularization methods in Hilbert scales, Numer. Math. 117 (2011), 555-579.

17. - Implicit iteration methods in Hilbert scales under general smoothness conditions, Inv. Prob. 27 (2011), doi:10.1088/0266-5611/27/4/045012.

18. A.G. Ramm, Inverse problems, Mathematical and analytical techniques with applications to engineering, Springer, New York, 2005.

19. A.G. Ramm, A.B. Smirnova and A. Favini, Continuous modified Newton'stype method for nonlinear operator equations, Ann. Mat. Pura Appl. 182 (2003), $37-52$.

20. E.V. Semenova, Lavrentiev regularization and balancing principle for solving ill-posed problems with monotone operators, Comp. Meth. Appl. Math. 4 (2010), 444-454.

21. M.E. Shobha, I.K. Argyros and S. George, Newton-type iterative methods for nonlinear ill-posed Hammerstein-type equations, Appl. Math., to appear.

22. U. Tautenhahn, On a general regularization scheme for non-linear ill-posed problems: II. Regularization in Hilbert scales, Inv. Prob. 14 (1998), 1607-1616.

23. - On the method of Lavrentiev regularization for nonlinear ill-posed problems, Inv. Prob. 18 (2002), 191-207.

24. — Error estimate for regularization methods in Hilbert scales, SIAM J. Numer. Anal. 33 (1996), 2120-2130.

Department of Mathematical and Computational Sciences, National Institute of Technology Karnataka, Surathkal-575 025, India

Email address: shobha.me@gmail.com

Department of Mathematical and Computational Sciences, National Institute of Technology Karnataka, Surathkal-575 025, India

Email address: sgeorge@nitk.ac.in

Department of Mathematics, Goa University, Goa-403 206, India

Email address: kunha@unigoa.ac.in 